\title{
Biologists tackle cells' identity crisis
}

\section{DNA fingerprinting scheme aims to make sure researchers are working on the right cells.}

Ever since biologists learned how to grow human cells in culture half a century ago, the cells have been plagued by a problem of identity: many commonly used cell lines are not actually what researchers think they are.

Cell-line misidentification has led to mistakes in the literature, misguided research based on those results and millions wasted in grant money. Last year, Nature described the situation as a scandal ${ }^{1}$.

But a universal system for determining the identity of cell lines may now be in view. Next month, a working group led by the American Type Culture Collection (ATCC), a nonprofit biological repository based in Manassas,

Virginia, that stores 3,600 cell lines from more than 150 species, plans to unveil standardized protocols for verifying the identity of cultured cells using DNA fingerprinting. Labs worldwide - including repositories such as the ATCC itself - would use the protocols to determine whether a breast-cancer line, for instance, did come from breast tissue. The group also plans to create a public database, which the National Center for Biotechnology Information in Bethesda, Maryland, has agreed to host, to store DNA profiles of validated lines, allowing researchers to compare their own cell cultures with the ATCC's reference lines.

"I really think it's fantastic progress," says Rolf König, director of the Tissue Culture Core Facility at the University of Texas Medical Branch in Galveston.

Misidentification can happen when faster-growing cells contaminate cultures of slowergrowing cells in the same lab, or when researchers simply mislabel a specimen. One particularly robust cell line called HeLa, the first human cell line grown in the lab, has contaminated dozens of other lines without researchers' knowledge ${ }^{2}$, and there are many other examples where melanoma cells and ovarian cells, for example, have been mistaken for breast cells. In this month's Nature Reviews Cancer $^{3}$, the ATCC consortium notes that one group has published around 20 papers since 1988 in which they incorrectly use a line called Int-407 as a model of normal intestinal cells.
"Without policing, many investigators may not be motivated to do the necessary tests."
The working group, composed of representatives from academia, government and industry, as well as from other cell repositories, advocates verifying cells' identities by comparing their DNA in regions where short stretches of three to five bases are repeated. Closely related cells are likely to have the same number of repeats; comparing these snippets at several different positions in the DNA sequence provides an overall estimate of relatedness.

Forensics applications, such as paternity testing and identifying crime victims, already use the technique. But cell lines often come from tumour tissue, in which DNA mutates at a higher rate than normal, making a $100 \%$ match between cells unlikely. Instead, the consortium suggests, cells that match at $75 \%$ or above can be considered to be the same. The group has now developed and tested a standardized procedure for extracting DNA from cells, doing the fingerprinting and interpreting the results.

Many researchers already use DNA fingerprinting to test their cell lines, notes Steve Oglesbee, director of the tissue-culture facility at the Lineberger Comprehensive Cancer Center of the University of North Carolina in Chapel Hill. The ATCC and other repositories have already established fingerprints for some of the most commonly used lines. "We're recommending that investigators authenticate from the beginning, and do it at least at the very end, and if they feel the need even during the work process," he says. Having

\section{HEALING CELLS}

Bone-marrow transplants cure obsessive-compulsive mice.

go.nature.com/TCIhZs

a universally accepted approach will allow different facilities to compare their cell lines with each other, he adds.

Fingerprinting has its limits, cautions Michael Johnson, a cancer researcher at Georgetown University in Washington DC. "Just because a cell fingerprints out as the same [as another cell] doesn't mean they will behave the same," he says, noting that a cell's properties can also be affected by the way it has been grown, the number of times it has been cultured anew and small genetic changes that wouldn't show up in a fingerprint test. One classic example, he notes, is an immortalized breast cell line called MCF10A, which can form organized hollow structures similar to those found in mammary tissue; MCF10A cells currently distributed by ATCC do not do this nearly as efficiently.

\section{Cell solution}

He worries that, useful though it would be, a database such as the one ATCC proposes "in some sense creates a false sense of security" about the "official version" of a cell line. Being able to keep track of a cell line's lineage where it was derived - could be as important as ascertaining its DNA fingerprint, he adds.

Others note that researchers will probably need an extra push to embrace the ATCC protocols. About half a dozen journals, including Wiley's International Journal of Cancer and journals published by the American Association for Cancer Research, have begun demanding that researchers authenticate their cell lines before they publish their work. And Nature has endorsed efforts to make verification easier and cheaper for researchers, pledging to require it once funders acknowledge the need and provide the necessary financial support ${ }^{1}$. "Without the policing by journal editors and granting agencies," says Gertrude Buehring, a virologist at the University of California, Berkeley, "many investigators may not be motivated to do the necessary tests to authenticate the cell lines used for their research."

Alla Katsnelson

Nature 457, 935-936 (2009).

2. Lucey, B. P. et al. Arch. Pathol. Lab. Med. 133, 1463-1467 (2009).

3. ATCC Standards Development Organization Workgroup AN-0002 Nature Rev. Cancer 10, 441-448 (2010). 